REF - ISSN 1808-0804 Vol. IX (3), 69 - 90, 2012.

\title{
FARMACOLOGIA DO DIABETES MELLITUS TIPO 2: ANTIDIABÉTICOS ORAIS, INSULINA E INOVAÇÕES TERAPÊUTICAS
}

PHARMACOLOGY OF DIABETES MELLITUS TYPE 2: ORAL ANTIDIABETIC PRODUCTS, INSULIN AND THERAPEUTIC INNOVATIONS

FARMACOLOGÍA DE LA DIABETES MELLITUS TIPO 2: ANTIDIABÉTICOS ORALES, INSULINA Y INNOVACIONES TERAPÉUTICAS

Vannessa Passos Lopes ${ }^{1}$, Manoelito Coelho dos Santos Júnior², Aníbal de Freitas Santos Júnior ${ }^{3 *}$, Amália Ivine Costa Santana ${ }^{4}$.

${ }^{1}$ Farmacêutica graduada pela Universidade Estadual de Feira de Santana (UEFS). Departamento de Saúde (DSAU). Feira de Santana - Bahia.

${ }^{2}$ Docente da Universidade Estadual de Feira de Santana (UEFS). Departamento de Saúde. Feira de Santana - Bahia.

${ }^{3}$ Departamento de Ciências da Vida (DCV) - Area de Farmacologia

${ }^{4}$ Enfermeira graduada pela Universidade Estadual de Feira de Santana (UEFS).

Departamento de Saúde (DSAU). Núcleo de Epidemiologia - UEFS. Feira de Santana Bahia.

*autor para correspondência: anibaljrr@uol.com.br

Recebido em 13/06/2012, Aceito em 28/12/2012

\section{RESUMO:}

O Diabetes mellitus do tipo 2 (DM-2) é uma desordem metabólica que apresenta a hiperglicemia como característica principal, caracterizado pela resistência à ação da 
Lopes, V.P.; Júnior, M.C.S.; Júnior, A.F.S.; Santana, A.I.C. Revista Eletrônica de Farmácia Vol. IX (4), 69 - 90, 2012.

insulina e disfunção das células $\beta$ pancreáticas. Este trabalho objetivou apresentar a farmacoterapia e os avanços no tratamento do DM-2. A metodologia empregada foi a pesquisa bibliográfica. O tratamento do DM-2 é multifatorial incluindo, primeiramente, educação e mudanças no estilo de vida e, posteriormente intervenção farmacológica. Os antidiabéticos orais constituem a primeira escolha para o tratamento do DM-2 não responsivo às medidas não farmacológicas isoladas e, classificam-se de acordo com o seu mecanismo de ação: 1) hipoglicemiantes orais ou secretagogos (sulfoniluréias e metiglinidas); 2) sensibilizadores da ação da insulina (tiazolidinodionas); 3) redutores da neoglicogênese (biguanidinas) e; 4) redutores da velocidade de absorção de glicídeos (inibidores da a-glicosidase). A busca por terapias com elevada eficácia e menos ocorrência de efeitos indesejáveis é ininterrupta e, novos fármacos tornam-se disponíveis, por exemplo, os incretinomiméticos; inibidores da Dipeptidil Peptidase 4 (DPP-IV) e análogos da amilina. Assim, devido a alta prevalência e as graves consequências associadas ao DM-2 tornou-se de ampla relevância compilar informações sobre os fármacos classicamente utilizados na terapêutica do DM-2, bem como os novos fármacos disponíveis o tratamento dessa doença.

PALAVRAS-CHAVE: Diabete Melito tipo 2; insulina; hiperglicemia; antidiabéticos.

\section{ABSTRACT:}

Diabetes mellitus type $2(\mathrm{DM}-2)$ is a metabolic disorder that presents the main characteristic of hyperglycemia, characterized by resistance to insulin action and dysfunction of pancreatic $\beta$ cells. This work aimed to present therapeutic advances for the treatment of DM-2. The methodology was a literature research. The treatment of DM2 is multifactorial including, first, education and changes in lifestyle and later pharmacological intervention. The antidiabetics are the first choice for treatment of DM-2 non-responsive to non-pharmacological measures isolated and classified according to their mechanism of action: 1) oral hypoglycemic drugs or secretagogues (sulfonylureas and metiglinides); 2) sensitizing action of insulin (thiazolidinediones); 3) reducing the gluconeogenesis (biguanide) and, 4) reducing the rate of absorption of glucose metabolism (inhibitors of a-glucosidase). The search for therapies with high efficiency and less occurrence of side effects is seamless and new drugs become available, for example, incretin mimetics; Dipeptidyl Peptidase-4 Inhibitors (DPP-IV) and analogues amylin. Thus, due to high prevalence and serious consequences associated with the DM-2 has become widely compile relevant information about the drugs classically used in the treatment of DM-2, as well as new drugs available for treating this disease.

KEYWORDS: Diabetes mellitus type 2, insulin, hyperglycemia, antidiabetics.

\section{RESUMEN:}


Lopes, V.P.; Júnior, M.C.S.; Júnior, A.F.S.; Santana, A.I.C. Revista Eletrônica de Farmácia Vol. IX (4), 69 - 90, 2012.

La diabetes mellitus tipo 2 (DM-2) es un trastorno metabólico que se presentan como característica principal de la hiperglucemia, caracteriza por la resistencia a la acción de la insulina y la disfunción pancreática $\beta$-células. Este estudio tuvo como objetivo presentar la farmacoterapia y los avances para el tratamiento de la DM-2. La metodología utilizada fue una búsqueda bibliográfica. El tratamiento de la DM-2 tiene un enfoque multifactorial, incluyendo, la educación en primer lugar, y cambios en el estilo de vida y tratamiento farmacológico más tarde. Los agentes hipoglucemiantes orales son la primera opción para el tratamiento de la DM-2 que no responden a medidas no farmacológicas solas, y se clasifican según su mecanismo de acción: 1) los agentes hipoglucemiantes orales o secretagogos (sulfonilureas y metiglinidas), 2) la acción de sensibilizadores de la insulina (tiazolidindionas), 3) la reducción de la gluconeogénesis (biguanidas) y 4) reducir la velocidad de absorción de glicídeos (a-glucosidasa inhibidores). La búsqueda de terapias con una alta eficiencia y menos efectos indeseables es continua y nuevos fármacos estén disponibles, por ejemplo, incretina; dipeptidilpeptidasa 4 (DPP-IV) y análogos amilina. Por lo tanto, debido a la alta prevalencia y graves consecuencias de la DM-2 se hizo muy recopilar información relevante acerca de los medicamentos clásicamente utilizados en el tratamiento de la DM-2, así como los nuevos fármacos disponibles para tratar esta enfermedad.

PALABRAS CLAVE: diabetes mellitus tipo 2, insulina, hiperglucemia, antidiabeticos.

\section{INTRODUÇÃO}

O crescimento populacional, o aumento da expectativa de vida e, principalmente, a crescente prevalência da obesidade associada ao sedentarismo e aos hábitos alimentares modernos, vem contribuindo para o aumento do número de indivíduos com Diabetes mellitus (DM). No Brasil, a população diabética, em 2000, alcançava 4,6 milhões de pessoas, sendo projetado para 2030 um número de 11,3 milhões de pessoas, o que corresponde a sexta posição na classificação dos países com maior prevalência dessa doença ${ }^{(1)}$.

O DM promove drásticas consequências sociais e econômicas devido à natureza crônica, à gravidade de suas complicações e aos meios necessários para controlá-la. Todos esses aspectos fazem do DM uma doença extremamente onerosa, não apenas para os indivíduos acometidos por esta patologia, mas também para os sistemas de saúde. (sugiro unir os parágrafos!!!) O DM configura-se como uma das causas mais importantes de mortalidade e morbidade na população em geral. Segundo dados da World Health Organization (WHO) ${ }^{(2)}$, em 2007, - DM foi responsável por $6 \%$ da mortalidade mundial. Vale ressaltar que o DM é uma enfermidade subdiagnosticada e que possui uma mortalidade subestimada, o que 
Lopes, V.P.; Júnior, M.C.S.; Júnior, A.F.S.; Santana, A.I.C. Revista Eletrônica de Farmácia Vol. IX (4), 69 - 90, 2012.

demonstra que sua magnitude é maior do que pode ser traduzida com esses dados estatísticos, acometendo um número maior de pessoas e sendo responsáveis por um maior número de óbitos.

Tendo em vista que o DM constitui um problema de saúde pública e que a forma mais incidente é o DM-2 (associado a cerca de $90 \%$ dos casos), justifica-se o desenvolvimento deste presente estudo, o qual teve como objetivo apresentar uma revisão de literatura referente à farmacoterapia e avanços terapêuticos disponíveis para o tratamento do Diabete Melito tipo 2.

DIABETES MELLITUS TIPO 2: FISIOPATOLOGIA E DIAGNÓSTICO

A homeostasia normal da glicose é rigidamente regulada por três processos relacionados: a produção de glicose no fígado; sua captação e utilização pelos tecidos periféricos, principalmente os músculos esqueléticos; e as ações da insulina e dos hormônios contrareguladores, incluindo o glucagon ${ }^{(3)}$. No DM-2, essa homeostasia é rompida e, como efeito final, tem-se a hiperglicemia. Esse tipo de DM é caracterizado pela resistência a ação da insulina e disfunção das células $\beta$ pancreáticas ${ }^{(4)}$. A resistência, em questão, refere-se à perda da sensibilidade dos tecidos à insulina, o que torna menos efetiva a estimulação para captação da glicose. Já a disfunção das células $\beta$ se manifesta pela secreção inadequada de insulina diante a resistência à mesma e a hiperglicemia. $\mathrm{Na}$ maioria dos casos a resistência à insulina é o evento primário, seguido de graus variados de disfunção das células $\beta^{(3)}$. Os principais eventos envolvidos no desenvolvimento do DM-2 são apresentados na Figura 01.

Figura 01 Fisiopatologia do Diabetes mellitus do tipo $2^{(5)}$.

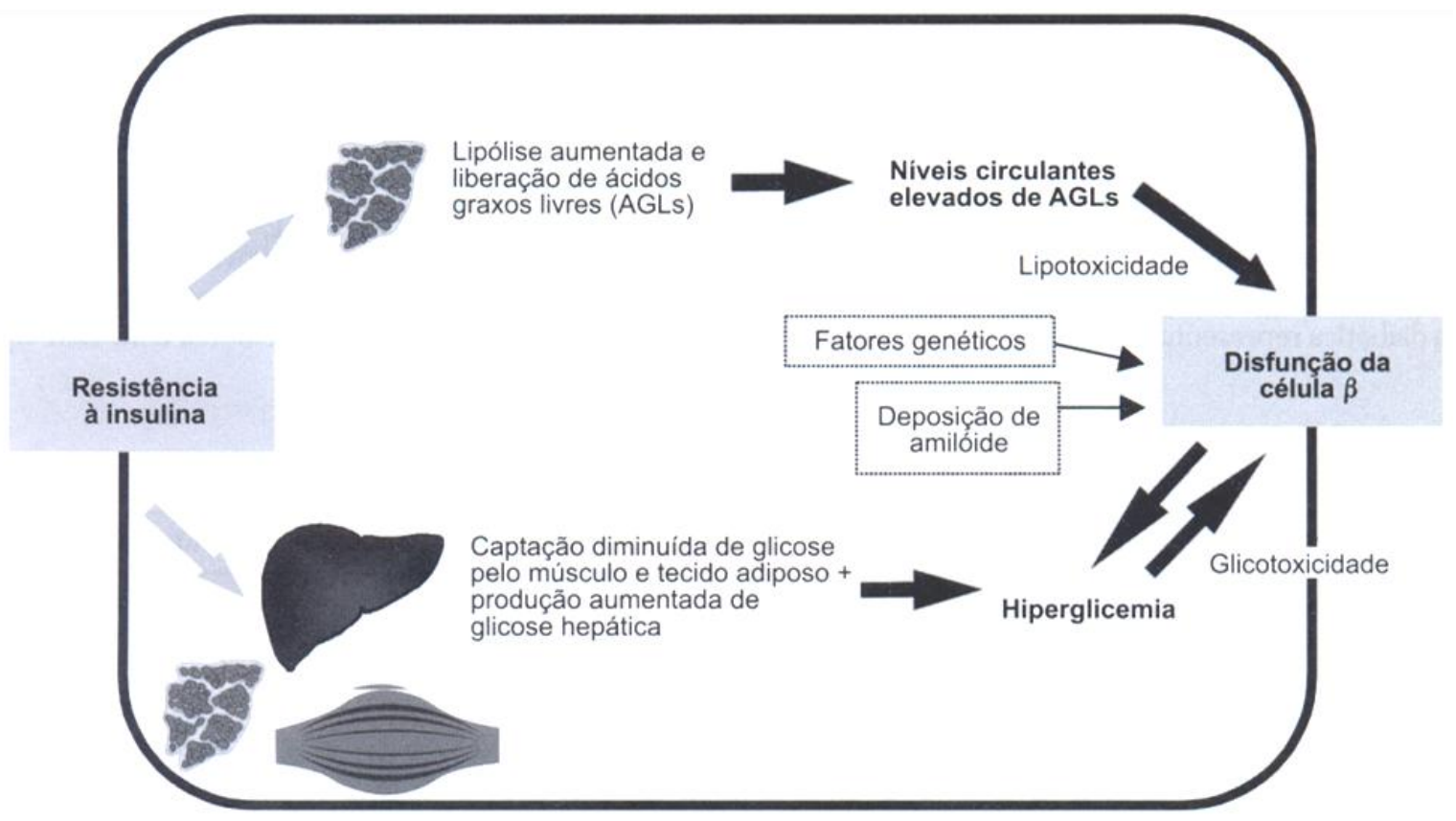


Lopes, V.P.; Júnior, M.C.S.; Júnior, A.F.S.; Santana, A.I.C. Revista Eletrônica de Farmácia Vol. IX (4), 69 - 90, 2012.

Tais eventos podem ser precipitados pela presença de certos fatores como: predisposição genética, obesidade, inatividade física e envelhecimento, que interferem ou na reserva funcional das células beta ou na sensibilidade tecidual à insulina ou em ambos os defeitos. É difícil definir, para cada paciente, qual a participação do componente de resistência à insulina e da deficiência insulínica, mas na maioria dos casos, as duas condições coexistem em proporções diferentes para diferentes pacientes $^{(4)}$. Segundo Ribeiro e colaboradores $^{(6)}$, o DM pode ser diagnosticado de três formas, segundo recomendações da American Diabete Association (ADA), Organização Mundial da Saúde (OMS) e Sociedade Brasileira de Diabete (SBD):

○Sintomas clássicos (poliúria, polidipsia e perda de peso) associado a uma glicemia ao acaso igual ou superior a 200 $\mathrm{mg} \mathrm{dL}^{-1}$. A glicemia ao acaso é definida como aquela realizada em qualquer horário, independente do tempo decorrido em relação à última refeição;

- Glicemia igual ou superior a $126 \mathrm{mg} \mathrm{dL}^{-1}$, em jejum. Definese jejum como ausência de ingestão calórica por pelo menos 8h;

- Glicemia após duas horas após sobrecarga oral de glicose igual ou superior a $200 \mathrm{mg} \mathrm{dL}^{-1}$ (teste de tolerância à glicose (TTG).

A realização do teste padronizado de tolerância à glicose deve ser precedida de pelo menos três dias de dieta irrestrita, contendo $150 \mathrm{~g}$ ou mais de carboidratos, atividade física habitual e no momento do teste não se deve fumar ou caminhar ${ }^{(6)}$. A Tabela 02 apresenta os critérios diagnósticos para as diferentes categorias.

\begin{tabular}{|c|c|c|c|}
\hline \multirow[b]{2}{*}{ Categoria } & \multicolumn{3}{|c|}{ Glicemia } \\
\hline & $\begin{array}{c}\text { Jejum (mg } \\
\left.\mathrm{dL}^{-1}\right)\end{array}$ & $\begin{array}{c}\text { TTG } 75 g-2 h \\
\left(m g ~ d L^{-1}\right)\end{array}$ & $\begin{array}{c}\text { Casual } \\
\left(\mathrm{mg} \mathrm{dL}^{-1}\right)\end{array}$ \\
\hline Normal & $<110$ & $<140$ & \\
\hline Tolerância a glicose diminuída & $\geq 110$ e $<126$ & $\geq 140$ e $<200$ & \\
\hline Diabete Melito & $\geq 126$ & $\geq 200$ & $\begin{array}{l}\geq 200 \text { com } \\
\text { sintomas }\end{array}$ \\
\hline
\end{tabular}


Lopes, V.P.; Júnior, M.C.S.; Júnior, A.F.S.; Santana, A.I.C. Revista Eletrônica de Farmácia Vol. IX (4), 69 - 90, 2012.

O diagnóstico, numa pessoa assintomática, nunca deve ser feito com base em um único valor anormal de glicemia. Para o doente assintomático é essencial, pelo menos, mais um teste laboratorial com resultado positivo, quer em jejum, ao acaso ou através do TTG. Se estes testes não permitirem confirmação do diagnóstico de DM, é aconselhável manter vigilância e fazer novos testes periodicamente até que $o$ diagnóstico seja claro( ${ }^{(8)}$.

\section{TRATAMENTO FARMACOLÓGICO} DO DIABETES MELLITUS DO TIPO 2

O DM é uma enfermidade heterogênea, que compreende diversas alterações metabólicas associadas à hiperglicemia. Por isso, seu tratamento requer uma abordagem ampla e multifatorial, desde educação e mudanças no estilo de vida que incluem a suspensão do fumo, aumento da atividade física e reorganização dos hábitos alimentares até intervenções farmacológicas. Nesse sentido, a participação do paciente é fundamental para o sucesso do tratamento ${ }^{(6,9)}$. Os

objetivos do tratamento do DM-2 incluem trazer o paciente o mais próximo possível da normalidade, no que se refere ao metabolismo de carboidratos, lipídios e proteínas e, consequentemente, compensação dos sintomas clínicos; além da prevenção das complicações metabólicas agudas: coma hiperosmolar e hipoglicemia e

$$
\begin{aligned}
& \text { prevenção das complicações } \\
& \text { crônicas }^{(6,11)} \text {. }
\end{aligned}
$$$$
\text { Na prática clínica, a avaliação do }
$$
controle glicêmico é feita através da utilização de dois recursos laboratoriais: os testes de glicemia e os testes de Hemoglobina Glicada $\left(\mathrm{HbA}_{1 \mathrm{c}}\right)^{(12)}$. Os testes de glicemia refletem o nível glicêmico atual e instantâneo, no momento exato do exame, enquanto os testes de $\mathrm{HbA}_{1 \mathrm{C}}$, tendo em vista que os eritrócitos têm um tempo de vida de aproximadamente 120 dias, refletem a glicemia média dos últimos dois a três meses antes do exame. A quantidade de glicose ligada à hemoglobina é diretamente proporcional à concentração média de glicose no sangue e ao tempo de exposição da hemoglobina a glicose. Assim, quanto mais inadequado for 0 controle glicêmico mais alta a $\mathrm{HBA}_{1 c}{ }^{(13)}$.

Quando o paciente com DM-2 não responde ou deixa de fazer adequadamente as medidas nãomedicamentosas, deve ser iniciada a farmacoterapia ${ }^{(7)}$. Os antidiabéticos orais constituem a primeira escolha para o tratamento do DM-2 não-responsivo às medidas não-farmacológicas isoladas, uma vez que promovem, com controle glicêmico, redução na incidência de complicações, têm boa aceitação pelos pacientes e são fáceis de ser administrados $^{(14)}$.Os antidiabéticos orais classificam-se de acordo com o seu mecanismo de ação em ${ }^{(5,15-16)}$ :

1) hipoglicemiantes orais propriamente ditos ou 
Lopes, V.P.; Júnior, M.C.S.; Júnior, A.F.S.; Santana, A.I.C. Revista Eletrônica de Farmácia Vol. IX (4), 69 - 90, 2012.

secretagogos, que incluem as sulfoniluréias e as metiglinidas, que estimulam as células $\beta$ e aumenta a insulinemia;

2) sensibilizadores da ação da insulina: tiazolidinodionas, os quais possuem ação periférica melhorando a ação insulínica e a captação de glicose pelas células;

3) redutores da neoglicogênese: biguanidinas, os quais reduzem a glicemia principalmente pela redução da formação de glicose hepática;

4) redutores da velocidade de absorção de glicídeos: inibidores da a-glicosidase, os quais retardam a absorção de carboidratos, fazendo diminuir a glicemia pós-prandial.

O Quadro 01 apresenta as classes de antidiabéticos orais disponíveis no Brasil e suas principais características.

Quadro 01 Antidiabéticos orais disponíveis no Brasil ${ }^{(7)}$

\begin{tabular}{|c|c|c|c|c|}
\hline Medicamento & $\begin{array}{c}\text { Mecanismo de } \\
\text { ação }\end{array}$ & $\begin{array}{c}\text { Contra- } \\
\text { indicações }\end{array}$ & $\begin{array}{c}\text { Efeitos } \\
\text { indesejáveis }\end{array}$ & $\begin{array}{c}\text { Outros efeitos } \\
\text { benéficos }\end{array}$ \\
\hline Sulfoniluréia & $\begin{array}{l}\text { Aumento da } \\
\text { secreção de } \\
\text { insulina }\end{array}$ & $\begin{array}{c}\text { Gravidez, } \\
\text { lactação, } \\
\text { insuficiência } \\
\text { renal e hepática } \\
\text { greve }\end{array}$ & $\begin{array}{l}\text { Hipoglicemia e } \\
\text { ganho de peso }\end{array}$ & \\
\hline Metiglinida & $\begin{array}{l}\text { Aumento da } \\
\text { secreção de } \\
\text { insulina }\end{array}$ & $\begin{array}{c}\text { Gravidez, } \\
\text { lactação, } \\
\text { insuficiência } \\
\text { renal e hepática } \\
\text { grave }\end{array}$ & $\begin{array}{c}\text { Hipoglicemia } \\
\text { leve e ganho } \\
\text { de peso } \\
\text { discreto }\end{array}$ & \\
\hline Biguadina & $\begin{array}{c}\text { Reduz a } \\
\text { produção de } \\
\text { glicose com } \\
\text { menor ação } \\
\text { sensibilizadora } \\
\text { da ação da } \\
\text { insulina }\end{array}$ & $\begin{array}{c}\text { Gravidez, } \\
\text { lactação, } \\
\text { insuficiência } \\
\text { renal, cardíaca, } \\
\text { hepática, } \\
\text { pulmonar e } \\
\text { risco } \\
\text { aumentado de } \\
\text { acidose láctica }\end{array}$ & $\begin{array}{c}\text { Desconforto } \\
\text { abdominal, } \\
\text { diarréia e } \\
\text { náuseas }\end{array}$ & $\begin{array}{l}\text { Diminuição de } \\
\text { eventos } \\
\text { cardiovasculares, } \\
\text { melhora do perfil } \\
\text { lipídico e } \\
\text { diminuição do } \\
\text { peso }\end{array}$ \\
\hline $\begin{array}{l}\text { Inibidores da } \\
\text { a-glicosidase }\end{array}$ & $\begin{array}{l}\text { Retardo da } \\
\text { absorção de }\end{array}$ & $\begin{array}{l}\text { Gravidez, } \\
\text { lactação, }\end{array}$ & $\begin{array}{l}\text { Desconforto } \\
\text { abdominal, }\end{array}$ & $\begin{array}{l}\text { Diminuição de } \\
\text { eventos }\end{array}$ \\
\hline
\end{tabular}


Lopes, V.P.; Júnior, M.C.S.; Júnior, A.F.S.; Santana, A.I.C. Revista Eletrônica de Farmácia Vol. IX (4), 69 - 90, 2012.

\begin{tabular}{|c|c|c|c|c|}
\hline & carboidratos & $\begin{array}{c}\text { doença } \\
\text { intestinal } \\
\text { inflamatória e } \\
\text { grave } \\
\text { insuficiência } \\
\text { hepática e renal }\end{array}$ & $\begin{array}{l}\text { diarréia e } \\
\text { flatulência }\end{array}$ & $\begin{array}{c}\text { cardiovasculares } \\
\text { e melhora do } \\
\text { perfil lipídico }\end{array}$ \\
\hline Glitazonas & $\begin{array}{l}\text { Aumento da } \\
\text { sensibilidade a } \\
\text { insulina em } \\
\text { músculos, } \\
\text { hepatócitos e } \\
\text { adipócitos }\end{array}$ & $\begin{array}{c}\text { Gravidez, } \\
\text { lactação, } \\
\text { insuficiência } \\
\text { hepática e } \\
\text { insuficiência } \\
\text { cardíaca das } \\
\text { classes III e IV }\end{array}$ & $\begin{array}{c}\text { Edema, anemia } \\
\text { e ganho de } \\
\text { peso }\end{array}$ & $\begin{array}{l}\text { Melhora do perfil } \\
\text { lipídico, redução } \\
\text { da gordura } \\
\text { hepática }\end{array}$ \\
\hline
\end{tabular}

\section{Sulfoniluréias}

As sulfoniluréias são os medicamentos mais antigos para 0 tratamento do DM-2 e, ainda, são amplamente prescritos. A principal ação das sulfoniluréias é exercida sobre as células $\beta$ das ilhotas pancreáticas e consiste em aumentar a secreção de insulina. Adicionalmente a este efeito, os medicamentos, dessa classe, reduzem os níveis séricos de glucagon ${ }^{(17-18)}$. É importante salientar que esses fármacos reduzem a glicemia em especial pelo estímulo à secreção pancreática de insulina, e requerem, portanto, células $\beta$ funcionantes para que possam atuar. As sulfoniluréias são classificadas de acordo com sua potência e época de surgimento como de primeira e segunda geração. As drogas consideradas de primeira geração incluem: tolbutamida, aceto-hexamida e clorpropamida, mas só a clorpropramida é comercializada no Brasil. As demais sulfoniluréias (glibenclamida, glicazida, glipizida e glimepirida) são classificadas como de segunda geração ${ }^{(5,19)}$.

As sulfoniluréias estão, particularmente, indicadas para pacientes com diabete não-obesos ou quando há perda de peso e na fase inicial da doença, visto que a evolução da doença está relacionada a uma maior disfunção das células $\beta$ e, consequentemente, uma ineficácia desses fármacos. Os pacientes obesos, por sua vez, respondem mal às sulfoniluréias, possivelmente como decorrência da resistência à insulina que costuma acompanhar a obesidade ${ }^{(7,19)}$.

Os fármacos dessa classe estabelecem uma resposta terapêutica satisfatória em cerca de $25 \%$ dos indivíduos com DM-2. Entretanto, cerca de $75 \%$ dos pacientes com DM-2 tratado com uma sulfoniluréia não irá alcançar o efeito desejado e exigirá, além da sulfoniluréia, um segundo agente oral(20). Em geral, observa-se um decréscimo de 
60-70 $\mathrm{mg} \mathrm{dL}^{-1}$ na glicemia em jejum e de 1 a $2 \%$ na $\mathrm{HbA}_{1 \mathrm{c}}$ em pacientes com níveis de glicose plasmática acima de $200 \mathrm{mg} \mathrm{dL}^{-1(5,7)}$. Com o tempo a sua eficácia começa a declinar, caracterizando a falência secundária pancreática, isto é, a incapacidade de manter uma resposta satisfatória à terapia com as sulfoniluréias, a longo prazo, continua sendo um problema desconcertante no tratamento do DM-2 com esses fármacos. Alguns fatores podem contribuir para essa falha secundária como, por exemplo, a diminuição progressiva das células $\beta, 0$ declínio da massa corporal magra ou o acúmulo de gordura e o sedentarismo $^{(17)}$. De um modo geral, a freqüência da falência secundária de cerca de $4 \%$ ao ano ${ }^{(5)}$.

\section{Metiglinidas (Glinidas)}

Atualmente, existem dois compostos disponíveis dessa classe: a repaglinida, derivada do ácido benzóico e a nateglinida que é derivado do aminoácido D-fenilalaninaa ${ }^{(14,21)}$. Esses fármacos apresentam a mesma ação secretagoga das sulfoniluréias e com rápido início de ação; a nateglinida começa a agir após 4 minutos da sua administração e sua ação dura duas horas, já a repaglinida apresenta início de ação após 10 minutos e duração da ação de 3-5 horas $^{(5,15)}$.

As metiglinidas são indicadas no tratamento inicial de pacientes com DM2, quando a hiperglicemia pós-prandial pode ser a alteração principal e não é possível controlá-la apenas com medidas não-farmacológicas ${ }^{(6)}$. Podem ser usadas na insuficiência renal ou hepática leve ou moderada, neste caso com pequenos ajustes de dose ${ }^{(15)}$.

As indicações dos fármacos dessa classe são muito semelhantes às indicações das sulfoniluréias. Portanto, podem ser utilizados como alternativa às sulfoniluréias em pacientes intolerantes a estes fármacos, alérgicos a enxofre, em idosos e, quando houver predomínio de hiperglicemia pós-prandial ${ }^{(17,22)}$. Quando comparadas com as sulfoniluréias, as metiglinidas apresentam um início de ação mais rápido, possibilitando o controle mais efetivo da hiperglicemia pós-prandial; possuem um tempo de ação curto estando, portanto, menos associadas a hipoglicemia grave e; causam menor ganho de peso. Entretanto, são menos eficazes no controle da $\mathrm{HbA}_{1 c}$ e da glicemia em jejum $^{(5,15)}$.

\section{Biguanidinas}

Os dois principais representantes desse grupo são a metformina e a fenformina, entretanto esta última foi retirada do mercado no Brasil, uma vez que leva a um maior risco de acidose láctica quando comparado à metformina ${ }^{(5)}$. Os pacientes com DM-2 apresentam um grau considerável de menor hiperglicemia em jejum, bem como níveis menores de glicemia pósprandial após a administração da 
Lopes, V.P.; Júnior, M.C.S.; Júnior, A.F.S.; Santana, A.I.C. Revista Eletrônica de Farmácia Vol. IX (4), 69 - 90, 2012.

metformina ${ }^{(17)}$. Como monoterapia, a metformina reduz a glicemia de jejum em 20 a 30\%, a glicemia pós-prandial em 30 a $40 \%$ e a $\mathrm{HbA}_{1 \mathrm{c}}$ em 1 a $2 \%{ }^{(5,21)}$.

A metformina é indicada a pacientes cuja hiperglicemia está relacionada com uma ação ineficaz da insulina, ou seja, a uma síndrome de resistência insulínica. Como se trata de um agente poupador de insulina, que não aumenta o peso corporal, é particularmente indicada no tratamento inicial dos pacientes obesos, de modo usual, é a primeira escolha no tratamento do indivíduo obeso com DM2, mas também pode ser utilizadas em pacientes não-obesos ${ }^{(5,6,17,19,21)}$.

A metformina é indicada também no tratamento da Síndrome dos Ovários Policísticos (SOP), esteato-hepatite nãoalcoólica e em pacientes com tolerância diminuída à glicose a fim de prevenir o surgimento do DM-2. O uso da metformina induz melhoras no perfil lipídico, caracterizadas pela redução nos níveis de triglicerídeos e Lipoproteína de Baixa Densidade (LDL) em cerca de 10 a $15 \%$, enquanto os níveis de colesterol HDL não se alteram ou aumentam discretamente ${ }^{(20)}$; esses efeitos são benéficos para a redução dos riscos de doenças cardiovasculares e mortalidade relacionada ao diabete. A metformina é o único agente terapêutico que reduz os

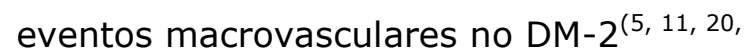
22, 23).

\section{Tiazolidinodionas (Glitazonas)}

As duas tiazolidinodionas disponíveis, à princípio, no Brasil foram a rosiglitazona e a pioglitazona. Em 2010, a rosiglitazona teve sua comercialização proibida no país, após a identificação da relação benefício-risco desfavorável, pela alta probabilidade de doenças isquêmicas em usuários deste medicamento. Um terceiro composto dessa classe, a troglitazona, também, foi retirada do mercado em virtude a sua hepatotoxicidade ${ }^{(6,20)}$. Esses fármacos mostram-se eficientes em pacientes obesos e não-obesos, sendo eficazes em $70 \%$ dos usuários iniciais. As glitazonas reduzem a glicemia em torno de $20 \%$ e a $\mathrm{HbA}_{1 \mathrm{c}}$ em 1 a 1,5\% e apresentam bom resultado na prevenção da progressão para DM-2, como também, são utilizadas como alternativa à metformina em pacientes com SOP ou esteato-hepatite não-alcoólica. Existem também evidências de que elas podem ter um efeito benéfico na preservação da função $\beta$, pela inibição da lipotoxicidade e glicotoxicidade ${ }^{(5,20)}$.

As tiazolidinodionas apresentam um custo mais elevado quando comparadas com a metformina, entretanto são vantajosas por não apresentarem predisposição a acidose láctica, causarem menos efeitos gastrintestinais, além de oferecerem a possibilidade da sua administração em pacientes com insuficiência renal leve a moderada. As glitazonas podem ter um efeito benéfico sobre o perfil lipídico, reduzindo $o$ colesterol total e 
Lopes, V.P.; Júnior, M.C.S.; Júnior, A.F.S.; Santana, A.I.C. Revista Eletrônica de Farmácia Vol. IX (4), 69 - 90, 2012.

triglicerídeos (10 a $20 \%$ ) e elevando o colesterol HDL em cerca de 10\%. Esses fármacos podem também aumentar os níveis de LDL em até $12 \%$ entretanto, predominando a elevação de partículas com maior poder de flutuação e menos susceptíveis a oxidação e, portanto, menos aterogênica ${ }^{(21-23)}$.

\section{Inibidores da a-glicosidase}

Os inibidores da a-glicosidase incluem: arcabose, coglibose e miglitol, entretanto somente a arcabose é comercializado no $\mathrm{Brasil}^{(7)}$. A monoterapia com arcabose está relacionada a uma pequena redução nos níveis de $\mathrm{HbA}_{1 \mathrm{c}}(0,5-1 \%)$ e a um declínio de 20-25 mg dL ${ }^{-1}$ nos níveis de glicose em jejum, sendo seu efeito mais pronunciado em pacientes com alta ingestão de carboidratos ${ }^{(7,21-23)}$.

Devido ao seu modesto efeito redutor da glicemia e $\mathrm{HbA}_{1 \mathrm{c}}$, bem como seus efeitos colaterais gastrintestinais, 0 emprego dessa droga, apesar de poder ser utilizada como terapia inicial do DM2 , é mais indicado para situações especiais incluindo: hiperglicemia predominantemente pós-prandial; pacientes idosos, não-obesos, com hipoglicemias freqüentes ao usarem sulfoniluréias e glinidas; como terapia adicional combinado com outros antidiabéticos oral ${ }^{(5)}$. Esse fármaco também pode ser útil em pacientes com tolerância alterada à glicose impedindo o a evolução para o DM-2, estando ainda ligados a restauração da função das células $\beta^{(17,21)}$.

\section{Insulina no Tratamento do DM-}

2

A insulinoterapia deve ser considerada em diabéticos tipo 2 quando, apesar do tratamento com agentes antidiabéticos orais, uma ou mais das seguintes situações estiverem presentes:

1) pacientes muito sintomáticos;

2) HbA1c > 7\%; surgimento de disfunção hepática e renal grave. É, também, indicada temporariamente durante a gestação e doenças agudas como, por exemplo, sepse e infarto agudo do miocárdio ou complicações agudas hiperglicêmicas (estado hiperglicêmico hiperosmolar não-cetótico e cetoacidose diabética) ${ }^{(5,24)}$.

Além disso, deve ser considerada também na ocasião do diagnóstico, quando os níveis de glicose estiverem muito elevados (275 - $300 \mathrm{mg} \mathrm{dL}^{-1}$ ). A insulinoterapia deve ser individualizada, principalmente, de acordo com o grau de hiperglicemia e os momentos em que esta ocorre ${ }^{(5,24)}$.

As insulinas disponíveis têm origem suína, bovino-suína (mista) e humana, além dos análogos e são classificadas de acordo com a farmacocinética em: de ação rápida, o único representante deste grupo é a Insulina Regular (IR); ultrarápida, representadas pelos análogos Lispro, Aspart e Glulisina, intermediária, que incluem a Neutral Protamine 
Lopes, V.P.; Júnior, M.C.S.; Júnior, A.F.S.; Santana, A.I.C. Revista Eletrônica de Farmácia Vol. IX (4), 69 - 90, 2012.

Hagedorn (NPH) e Lenta e; lenta, que engloba a Ultralenta, a Glargina e Detemir $^{(25)}$.

A insulina de ação rápida, IR, tem seu início de ação, após injeção subcutânea, entre 30 e 60 minutos, seu efeito máximo é observado em 2 a 3 horas e apresenta duração efetiva de 810 horas. A variabilidade inter e intraindividual é baixa, o que contribui para a previsibilidade de seus efeitos terapêuticos. A principal indicação é o controle da glicemia pós-prandial. A insulina regular deve ser administrada 30 a 60 minutos antes das refeições, para evitar o desencontro entre o seu pico de ação e a absorção de carboidratos da refeição ( 1 a 2 horas após o início da refeição) ${ }^{(26)}$.

A insulina de ação ultra-rápida tem início de ação mais rápido e duração mais curta em comparação a IR com a qual compartilha a mesma indicação. Assim, pode ser aplicada logo antes das refeições, enquanto a IR requer administração 30 minutos antes. Em uso subcutâneo, ambos têm início entre 5 e 15 minutos com pico entre 1 e 2 horas e término de ação entre 4 a 6 horas. Apresenta um perfil farmacocinético que se aproxima da secreção fisiológica de insulina pelas células $\beta$ em resposta a alimentação rica em carboidratos em indivíduos não diabéticos. Dessa forma, propiciam melhor controle da glicemia pós-prandial e menor risco de hipoglicemias quando comparadas a IR. Pode ser utilizada por via intravenosa ou intramuscular e pode ser associada a uma insulina de ação intermediária ou longa para melhor mimetizar a secreção hormonal diária normal ${ }^{(5,19,25-26)}$.

A NPH, insulina de ação intermediária, após a injeção subcutânea, tem início de ação em 2-4 horas, pico de ação de 4-10 horas e duração efetiva de 12-18 horas. Já a insulina lenta, que também é uma insulina de ação intermediária, apresenta a duração efetiva de até 20 horas. A principal finalidade das insulinas NPH e lenta é o controle glicêmico durante a noite e madrugada, bem como, nos períodos interprandiais através da suspensão da glicogenólise e gliconeogênese hepática ${ }^{(5,25)}$.

A insulina de ação lenta tem como principal representante a Glargina, cujo início de ação ocorre dentro de 2 a 4 horas, após injeção subcutânea. Tem duração uniforme durante aproximadamente 24 horas, sem picos, com variabilidade inter e intra-individual baixa, menor do que a encontrada com a insulina NPH. É a insulina que mais se aproxima do perfil insulínico obtido com a infusão subcutânea contínua (bomba de insulina). A insulina ultralenta é pouco utilizada devido a sua variabilidade inter e intra-individual o que dificulta a padronização de doses e consequentemente gera resultados terapêuticos pouco satisfatórios. A insulina Detemir apresenta uma ação lenta, nas doses usuais, de até 20 horas. A principal indicação das insulinas 
Lopes, V.P.; Júnior, M.C.S.; Júnior, A.F.S.; Santana, A.I.C. Revista Eletrônica de Farmácia Vol. IX (4), 69 - 90, 2012.

Glargina e Detemir é prover a insulina basal. Portanto, é necessária a coadministração da IR, Lispro, Aspart ou Glulisina para evitar hiperglicemia pósprandial. Tem como vantagens sobre a $\mathrm{NPH}$ o fato de necessitarem apenas uma aplicação diária (no caso da Glargina), terem melhor previsibilidade de ação, ausência de picos e menores riscos de hipoglicemia, principalmente noturna ${ }^{(25)}$.

Os principais efeitos indesejáveis relacionados à insulinoterapia incluem: a hipoglicemia, sendo mais comum com a insulinoterapia intensiva, omissão de refeição, erro na dose da insulina, bem como excessiva atividade física e ingestão de bebidas alcoólicas; o ganho de peso e; as reações alérgicas, principalmente no local da injeção, caracterizadas por eritema, endurecimento, prurido ou sensação de queimor, mais presente no tratamento com a insulina animal, estando presente em menos de $1 \%$ dos pacientes tratados com insulina humana. A alergia sistêmica é rara, devido, principalmente, ao alto grau de pureza com que a insulina é fabricada $^{(25,27) \text {. }}$

Uma nova opção para a insulinoterapia é o advento da insulina inalada, que foi aprovada pelo Food and Drugs Adminsitartion (FDA) em 2006. Porém, existem relatos que a FDA não aprovou o uso da insulina em pó (Afrezza $®$ ) e, a insulina inalável Exubera $\AA$ teve sua comercialização interrompida pela Pfizer em 2007.Tratase de uma insulina de ação rápida em pó para administração de maneira inalável. Após a inalação a insulina é absorvida pelos pulmões atingindo, em seguida, a corrente sanguínea. Apresenta controle glicêmico semelhante ao observado com a administração subcutânea de uma insulina de ação rápida, sendo indicada à pacientes com DM-2 maiores de 18 anos que necessitem da insulina para atingir um controle glicêmico e deve ser administrada antes das refeições. Possui sua biodisponibilidade aumentada pelo tabagismo e diminuída pela asma. Os efeitos indesejáveis mais comuns são a tosse e a hipoglicemia ${ }^{(7,22,25)}$.

\section{NOVAS OPÇÕES PARA TRATAMENTO DO DM-2}

A busca por novas terapias para o DM-2 com elevada eficácia e menor ocorrência de efeitos indesejáveis é ininterrupta e facilita o propósito interdisciplinar e multiprofissional no tratamento da DM-2. Nesse contexto, há um incremento no desenvolvimento de novos fármacos abrangendo áreas anteriormente inexploradas da fisiopatologia do diabete e alguns destes já estão disponíveis no mercado como, por exemplo, os incretinomiméticos; inibidores da Dipeptidil Peptidase 4 (DPP-IV) e análogos da amilina.

\section{Incretinomiméticos}

e Inibidores da DPP-IV

Especificamente, essas terapias inovadoras no tratamento do DM-2 são baseadas nos efeitos glicorregulatórios 
Lopes, V.P.; Júnior, M.C.S.; Júnior, A.F.S.; Santana, A.I.C. Revista Eletrônica de Farmácia Vol. IX (4), 69 - 90, 2012.

das incretinas ${ }^{(22)}$. O conceito de incretina foi criado a partir de estudos que relataram maior resposta da insulina à glicose administrada por via oral, contra uma concentração equivalente de glicose intravenosa(28). Assim se presumiu que determinados hormônios gastrintestinais deveriam ter uma ação aditiva sobre a secreção de insulina estimulada pela glicose por via oral, esses hormônios foram denominados incretinas ${ }^{(29)}$.

As incretinas são secretadas pelas células endócrinas localizadas no epitélio do intestino. Existem dois hormônios principais: o Peptídeo-1 Semelhante ao Glucacon (GLP-1) e o Peptídeo Inibitório Gástrico $(\mathrm{GIP})^{(30)}$. Esses hormônios são secretados em resposta a nutrientes e estimulam a secreção de insulina glicose-dependente ${ }^{(31-32)}$. O GIP e o GLP1 possuem alguns efeitos divergentes, 0 GLP-1 retarda o esvaziamento gástrico, suprime a secreção de glucagon e aumenta a saciedade, diminuindo o apetite. Já o GIP acelera o esvaziamento gástrico e não afeta a secreção de glucagon ou a saciedade ${ }^{(33)}$.

No DM-2 a secreção do GIP é preservada, enquanto a do GLP-1 é diminuída cerca de 20 a 30\%. Além disso, os pacientes com DM-2 têm resposta insulinotrópica deficiente à administração exógena de GIP, mas têm resposta preservada ao GLP-1 exógeno. Portanto, o GLP-1 é a incretina mais importante na patogenia do DM-2. Cerca de $60 \%$ da secreção de insulina pós- prandial é devida a ação das incretinas ${ }^{(28-29)}$.

Um entendimento maior sobre a função das incretinas no DM-2 levou à pesquisa e desenvolvimento de fármacos que objetivam a ampliação da estimulação e da ação do GLP-1. O uso terapêutico do GLP-1 é inviabilizado por este possuir meia-vida plasmática extremamente curta, inferior a 3 minutos, devida à sua rápida inativação pela enzima DPP-IV. Para contornar essa limitação foram desenvolvidas duas estratégias: o desenvolvimento de incretinomiméticos (análogos do GLP-1 resistentes à inativação enzimática $e$ agonistas do receptor do GLP-1) e; a criação de agentes inibidores da DPP-IV. Tanto os agonistas quanto os análogos do GLP-1 apresentam o inconveniente de serem drogas injetáveis enquanto os inibidores da DPP-IV são ativos por via oral $^{(34-35)}$.

\section{Análogos do GLP-1}

O liraglutide é um análogo de longa duração do GLP-1, com 97\% de homologia, que tem a propriedade de ser mais resistente a ação da enzima DPPIV, que é responsável pela degradação do $\operatorname{GLP}-1^{(7,36)}$. A administração de liraglutide em ensaios clínicos tem se mostrado eficiente em melhorar o controle glicêmico de pacientes com DM2 com eficácia na redução tanto da hiperglicemia de jejum quanto da pósprandial $^{(37)}$. 
Lopes, V.P.; Júnior, M.C.S.; Júnior, A.F.S.; Santana, A.I.C. Revista Eletrônica de Farmácia Vol. IX (4), 69 - 90, 2012.

Foi evidenciado em um recente estudo desenvolvido com1091 pacientes com DM-2 durante 26 semanas, tratados aleatoriamente $(2: 2: 2: 1: 2)$ com: Liraglutide $(0.6 ; 1,2$; ou $1,8 \mathrm{mg} / \mathrm{dia})$; placebo e; glimepirida ( $4 \mathrm{mg} / \mathrm{dia}$ ), todos os tratamentos foram em terapia de combinação com metformina $(1 \mathrm{~g}$, duas vezes por dia), que os grupos recebendo Liraglutide (1,2 e 1,6 mg) e glimepirida (4mg) apresentaram uma redução da $\mathrm{HbA}_{1 \mathrm{c}}$ de $1 \%$, os pacientes tratados com Liraglutide $(0,6 \mathrm{mg})$ reduziu o $\mathrm{HbA}_{1 \mathrm{c}} \mathrm{em}$ $0,7 \%$, enquanto o grupo placebo apresentou uma elevação de $0,1 \%$. A liraglutide apresentou incidência de hipoglicemia comparável ao placebo e náusea foi relatada por 11-19\%, essa incidência diminuiu ao longo do tempo ${ }^{(36)}$.

Em estudos de fase II, a liraglutide obteve reduções de $0,8 \%$ na hemoglobina glicada em comparação com o placebo ${ }^{(37)}$. Estudos adicionais são necessários para determinar seu pleno potencial terapêutico, mas estudos atuais apontam esse fármaco como promissor para o tratamento do DM-2. Liraglutide está atualmente em análise pela FDA para o tratamento do DM- $2^{(38)}$.

\section{Agonistas do GLP-1}

O fármaco representante dessa classe é a exenatida, um composto sintético análogo da exendina-4 (hormônio isolado da saliva do lagarto Gila monster). A exenatida mimetiza os efeitos do GLP-1 e compartilha com este
$50 \%$ de identidade da seqüência de aminoácidos. A exenatida é útil no tratamento do DM-2 pelo seu efeito redutor sobre as glicemias de jejum e pós-prandial. A exenatida foi aprovada nos Estados Unidos, pelo FDA, em maio de $2005^{(37,39)}$. Atualmente, está disponível também nos países membros da União Européia e partes da América Latina, incluindo o Brasil, onde passou a ser comercializado no primeiro semestre de $2008^{(31)}$.

Em ensaios clínicos o tratamento com exenatida resultou em significativas reduções significa nos níveis de $\mathrm{HbA}_{1 \mathrm{C}}$ variando de $0,77 \%$ a $1,1 \%{ }^{(31)}$. Observou-se, também, uma redução do peso corporal de 2 a $3 \mathrm{~kg}$ ao longo de seis meses ${ }^{(40)}$. A exenatida foi testada em associação com metformina e sulfoniluréias com segurança e eficácia. As associações com inibidores de aglicosidase e glinidas não foram testadas, mas devido ao modo de ação e alvo terapêutico específico sobre a glicemia pós-prandial, não foi considerada esta combinação. Existe uma base teórica para a combinação com glitazonas em função de mecanismos de ação diferentes e complementares, no entanto, não existem dados clínicos sobre esta combinação ${ }^{(41)}$.
Inibidores
da
DPP-IV 
Esses fármacos são uma nova classe de agentes antidiabéticos orais, devido à sua capacidade de prolongar a atividade do GLP-1 através da inibição da DPP-IV, consequentemente, os seus efeitos biológicos serão mais evidenciados $^{(42)}$. A DPP-IV é uma representante de uma família de enzimas existentes na circulação e na superfície de múltiplos tecidos, principalmente células epiteliais e endoteliais. Sua atividade consiste em clivar cadeias peptídicas nas quais está presente a prolina ou a alanina, como o segundo aminoácido a partir da extremidade N-terminal. A DPP-IV também está presente na membrana celular dos linfócitos $T$ ativados, coestimulando a molécula CD-45 na transdução do sinal. Nesta localização e com esta função a DPP-IV é conhecida como CD-26. Estudos recentes sugerem que a função enzimática de protease não é requerida para a função imunológica da CD-26, por conseguinte não se observa deficiência imunológica nos pacientes tratados com gliptinas. O perfil de segurança observado nos estudos clínicos sugere a existência de vias alternativas de degradação para outros peptídeos bioativos metabolizados pela DPP-IV, o que compensa a inativação dessa enzima( ${ }^{(34)}$.

Dois inibidores da DPP-IV, sitagliptina e a vildagliptina, já foram aprovados para o tratamento do DM-2 e estão disponíveis em diversos países, incluindo o Brasil. Têm demonstrado eficácia clínica em reduzir a excursão da glicemia pós-prandial através da melhora da função na célula $\beta$ com aumento da secreção de insulina pós-prandial e pela supressão da secreção de glucagon na presença de níveis glicêmicos elevados $^{(7,31)}$.

Os inibidores da DPP-IV são indicados para o tratamento do DM-2 em monoterapia ou em terapia combinada com a metformina ou glitazonas. Nestas condições, os estudos clínicos mostram que a incidência de hipoglicemia é comparável ao grupo placebo. A associação dos inibidores da DPP-IV com medicações secretagogas de insulina ou com a própria insulina, traz um risco potencial de hipoglicemias severas pela supressão simultânea do glucagon. Quando utilizados em monoterapia, os inibidores da DPP-IV podem levar a uma redução da $\mathrm{HbA}_{1 \mathrm{c}}$ entre $0,6 \%$ e 1,8\%. De um modo geral, como ocorre com os outros agentes orais, a redução é maior nos pacientes que têm hemoglobina glicada inicial acima de $9 \%{ }^{(34)}$.

\section{Análogo da amilina}

A amilina ou polipeptídio associado à ilhota é um peptídeo com 37 aminoácidos que é co-secretado com a insulina pelas células $\beta$ das ilhotas pancreáticas $^{(43)}$. A sua secreção é estimulada pela ingestão de alimentos, glucagon, GLP-1 e agonistas colinérgicos e inibida pela somatostatina e insulina. Suas ações são semelhantes às do GLP- 
Lopes, V.P.; Júnior, M.C.S.; Júnior, A.F.S.; Santana, A.I.C. Revista Eletrônica de Farmácia Vol. IX (4), 69 - 90, 2012.

1 , com a diferença de que não possuem nenhum poder secreção de insulina ${ }^{(44)}$.

Os receptores da amilina localizamse, principalmente, no SNC e estão relacionados com a regulação de muitos tecidos periféricos, tais como o pâncreas (inibe a secreção pós-prandial de glucagon e, portanto, evita a produção hepática de glicose) e o trato gastrintestinal (promove o atraso do esvaziamento gástrico). Além disso, atua sobre o centro da saciedade reduzindo a ingestão de alimentos ${ }^{(44-45)}$. Os níveis de amilina são deficientes no DM-1 e insuficiente no estado pós-prandial no DM-2.

O representante da classe dos análogos da amilina é a pramlintida, um análogo sintético do hormônio amilina $^{(22,40)}$. A pramlintida foi aprovada em 2005 nos EUA e Europa e, é indicada para 0 tratamento do $\mathrm{DM}-2$ com pacientes tratados com insulina e como adjuvante no tratamento do DM-1 $1^{(22,44-}$ 45). A $\mathrm{HbA}_{1 \mathrm{c}}$ foi reduzida entre 0,5 a $0,7 \%$ com a utilização deste medicamento $^{(40)}$.

\section{CONSIDERAÇÕES FINAIS}

O DM-2 constitui um dos mais sérios problemas de saúde pública da atualidade, sendo uma das principais enfermidades crônicas que acometem a população. Ademais, apresenta alto custo financeiro para o controle metabólico e tratamento de suas complicações.O objetivo principal do tratamento é adequar os níveis glicêmicos para o mais próximo possível da normalidade, de maneira rápida e eficiente, com o menor número de efeitos indesejáveis associados.

A busca por terapias com elevada eficácia e menor ocorrência de efeitos indesejáveis é constante e o conhecimento mais detalhado da fisiopatologia aliado a caracterização dos genes envolvidos no desenvolvimento dessa doença configura-se como um caminho promissor para a obtenção de uma terapia mais racional e específica. Nos últimos anos houve avanços importantes no conhecimento de novas estratégias de tratamento e várias classes de medicamentos foram introduzidas recentemente no mercado. Apesar dos avanços terapêuticos no tratamento do DM-2, o controle do nível glicêmico e das complicações crônicas ainda não são totalmente satisfatórios.

Diante disto, a equipe multiprofissional de saúde deve posicionar-se de forma interdisciplinar e ativa na orientação e educação do paciente diabético, no que diz respeito tanto a aspectos relacionados com a terapia farmacológica quanto à conduta adequada que deve ser seguida para alcançar o controle da doença, bem como prevenir o surgimento das suas complicações. 
Lopes, V.P.; Júnior, M.C.S.; Júnior, A.F.S.; Santana, A.I.C. Revista Eletrônica de Farmácia Vol. IX (4), 69 - 90, 2012.

\section{REFERÊNCIAS}

1. Wild S et al. Global prevalences of diabetes. Estimates for the year 2000 and Projections for 2030. Diabetes Care, v. 27, n. 5, p. 1047-1053, 2004.

2. WORLD HEALTH ORGANIZATION (WHO). Press Releases Diabetes Summit for SEA Region raises clarion call for partnerships. Geneva, 2008. Disponível em: < http://www.searo.who.int/en/Section1257/Section2181/Section2211/Section2521_1469 2.htm>. Acesso em: 03 jun. 2009.

3. Maitra A, Abbas AK. O Sistema endócrino. In: Robbins SL, Cotran RS, Kumar V. Patologia estrutural e funcional. 7. ed. Rio de Janeiro: Guanabara Koogan, 2005. p. 1207-1282.

4. Velloso L, Pimazoni Netto A. Fisiologia e fisiopatologia das células beta: implicações clínicas e terapêuticas, 2008. Disponível em: < http://www.diabetes ebook.org.br/capitulo/fisiologia-e-fisiopatologia-das-celulas-beta-implicacoes-clinicas-eterapeuticas/>. Acesso em: 20 jun. 2009.

5. Matheus et al. Tratamento farmacológico do Diabetes tipo 2. In Vilar L, Kater CE. Endocrinologia clínica. 3. ed. Rio de Janeiro: MEDSI, 2006. 573-592.

6. Ribeiro MRF et al. Diabetes Melito. In: Lopes AC, Amato Neto V. Tratado de clínica médica. São Paulo: Roca, 2006. p. 3570-3594.

7. Sociedade Brasileira de Diabetes (SBD). Diretrizes da Sociedade Brasileira de Diabetes. 2008. Disponível em: < http://www.diabetes.org.br/ educacao/docs/Diretrizes_SBD_2008_MAR_12.pdf>. Acesso em: 20 jul. 2009.

8. Sociedade Portuguesa de Diabetologia (SPD). Definição, Diagnóstico e Classificação da Diabetes Mellitus, 2009. Disponível em: < http://www.spd.pt/ index.php? option=com_content\&task=view\&id=58\&Itemid=30>. Acesso em: 08 jul. 2009.

9.

Sociedade Brasileira de Diabetes (SBD). Diagnóstico e classificação do diabete melito e tratamento do diabete melito do tipo 2. Consenso Brasileiro Sobre diabetes 2002. Rio de Janeiro: Diagraphic, 2003. 
10. Farhat FCLG, Iftoda DM, Santos PH. Interações entre Hipoglicemiantes Orais e Alimentos. Saúde em revista, Piracicaba, v. 9, n. 21: p. 57-62, 2007.

11. Freitas EV. Diabetes Mellitus. In: . Tratado de geriatria e gerontologia.

2. ed. Rio de Janeiro: Guanabara Koogan, 2006. p.776-790.

12.

SOCIEDADE BRASILEIRA DE

DIABETES (SBD). Tratamento e acompanhamento do diabete melito: Diretrizes da Sociedade Brasileira de Diabetes. Rio de Janeiro: Diagraphic, 2007.

13. Forti et al. Diabetes Mellitus: classificação e diagnóstico. in Vilar L, Kater CE. Endocrinologia clínica. 3. ed. Rio de Janeiro: MEDSI, 2006. p. 539-549.

14. Matos MCG, Branchtein L. O uso de antidiabéticos orais no paciente com diabetes mellitus e doença cardíaca. Revista da Sociedade de Cardiologia do Rio Grande do Sul, Porto Alegre, ano XV, n. 08, mai/jun/jul/ago, 2006.

15. Ramalho ACR, Lima ML. Insulina e antidiabéticos orais. In: Silva, P. Farmacologia. 7. ed. Rio de Janeiro: Guanabara Koogan, 2006. p. 805-823.

16. Araujo LMB, Britto MMS, Cruz TRC. Tratamento do diabetes mellitus do tipo 2: novas opções. Arquivos Brasileiros de Endocrinologia \& Metabologia, v. 44, n. 6, p. 509-518, dez. 2000.

17. Nolte MS, Karam JH. Hormônios pancreáticos e fármacos antidiabéticos. In: Katzung BG Farmacologia: básica e clínica. 9. ed. Rio de Janeiro; Guanabara Koogan, 2006. p. 579-597.

18. Rang HP et al. Farmacologia. Rio de Janeiro: Campus, 2004.

19. Branchtein L, Matos MCG. Antidiabéticos. In: Fuchs FD, Wannmacher L, Ferreira MBC. Farmacologia clínica: fundamentos da terapêutica racional. 3. ed. Rio de Janeiro: Guanabara Koogan, 2006. p. 832-844.

20. Defronzo RA. et al. Effects of Exenatide (Exendin-4) on Glycemic Control and Weight Over 30 Weeks in Metformin-Treated Patients With Type 2 Diabetes. Diabetes Care, v. 28, n. 5, p. 1092-1100, maio 2005. 
21. Marcondes JAM. Diabete melito: Fisiopatologia e tratamento. Revista da faculdade de ciências médicas de Sorocaba, Sorocaba, v. 5, n.1, p. 16-28, 2003.

22. González EO, Pérez MC, Gumpert SA. Actualización del tratamiento farmacológico de la diabetes mellitus tipo 2. Inf Ter Sist Nac Salud, v. 32, n. 1, p. 3-16. 2008.

23. Davis NS, Granner DK. Insulina, hipoglicemiantes orais e a farmacologia do pâncreas endócrino. In: Goodman \& Gilman: as bases farmacológicas da terapêutica. 10. ed. Rio de Janeiro: McGraw-Hill, p.1263-1289, 2005.

24. Young EC, Pires MLE, Lima MBC. Abordagem terapêutica no diabetes mellitus tipo 2. Cadernos Brasileiros de Medicina. v. XIV, n. 1,2,3,4, jan./dez. 2001. Disponível em: < http://www.unirio.br/ccbs/revista/cadbra2001/ endocrinologia.htm>. Acesso em: 21 jul. 2009.

25. Rassi N, Silva SC. Insulinoterapia no Diabetes tipo 1. In Vilar L, Kater CE. Endocrinologia clínica. 3. ed. Rio de Janeiro: MEDSI, 2006. p. 601-614.

26. Pires AC, Chacra AR. A evolução da insulinoterapia no diabetes melito tipo 1 . Arquivos Brasileiros de Endocrinologia \& Metabologia, São Paulo, v. 52, n. 2, mar. 2008.

27. Camata DG. Complicações locais na pele, relacionadas à aplicação de insulina. Revista Latino-Americana de Enfermagem, Ribeirão preto, v. 11, n.1, jan./fev. 2003.

28. Chacra AR. Efeito fisiológico das incretinas. Johns Hopkins Advanced Studies in Medicine, v. 6, n. 7B, p. 613-617, jul. 2006.

29. Talaulicar M. Sobre a incretina e a sua aplicação no tratamento da diabetes mellitus tipo 2. Revista Da Sociedade Portuguesa De Medicina Interna, v. 15, n. 31, p. 207-213, jul./set. 2008.

30. Tambascia MA. O complexo insulina/glucagon/incretinas e seu papel no controle glicêmico. Revista Brasileira de Medicina, n. 3, p. 8-10, 2007. Suplemento Especial. 
Lopes, V.P.; Júnior, M.C.S.; Júnior, A.F.S.; Santana, A.I.C. Revista Eletrônica de Farmácia Vol. IX (4), 69 - 90, 2012.

31. Davidson JA, Parente EB, Gross JL. Incretin Mimetics and Dipeptidyl Peptidase-4 Inhibitors: Innovative Treatment Therapies for Type 2 Diabetes. Arquivos Brasileiros de Endocrinologia \& Metabologia, v. 52, n. 6, p.1039-1049, 2008.

32. Farilla L. et al. Glucagon-like peptide 1 inhibits cell apoptosis and improves glucose responsiveness of freshly isolated human islets. Endocrinology, v. 144, n. 12, p. 5149-5158, 2003. Disponível em: < http://endo.endojournals.org/cgi/conten t/abstract/en.2003-0323v1 >. Acesso em: 28 jul. 2009.

33. Drucker DJ. Enhancing Incretin Action for theTreatment of Type 2 Diabetes. Diabetes Care, v. 26, n. 10, p. 2929-2940, out. 2003.

34. Eliaschewitz FG. Perfil terapêutico dos inibidores da enzima Dipeptidilpeptidase IV (DPP-IV). Revista Brasileira de Medicina, n.3, p. 11-15, 2007. Suplemento Especial.

35. Ahren B. et al. Inhibition of dipeptidyl peptidase-4 reduces glycemia, sustains insulin levels and reduces glucagon levels in type 2 diabetes. The Journal of Clinical Endocrinology \& Metabolism, v. 89, n. 5, p. 2078-2084, 2004.

36. Nauck M. et al. Efficacy and Safety Comparison of Liraglutide, Glimepiride, and Placebo, All in Combination With Metformin, in Type 2 Diabetes. Diabetes Care, v. 32, n. 1, p. 84-90, jan. 2009.

37. Geloneze B, Lamounier RN, Coelho OR. Hiperglicemia pós-prandial: tratamento do seu potencial aterogênico. Arquivos Brasileiros de Cardiologia, v. 87, p. 660-670, 2006.

38. Sakauye SD, Shah SA. Liraglutide: A human GLP-1 analogue for the treatment of type 2 diabetes. Formulary, n. 44, n. 5, p. 136-142, maio 2009. Disponível em: < http://formularyjournal.modernmedicine.com/formulary/Endocrinolo gy/Liraglutide-Ahuman-GLP-1-analogue-for-the-treatme/ArticleStandard/Article/det ail/596635>. Acesso em: 25 jul. 2010.

39. Edwards CMB. et al. Exendin-4 reduces fasting and postprandial glucose and decreases energy intake in healthy volunteers. Am J Physiol Endocrinol Metab, v. 281, p. 155-161, 2001. 
Lopes, V.P.; Júnior, M.C.S.; Júnior, A.F.S.; Santana, A.I.C. Revista Eletrônica de Farmácia Vol. IX (4), 69 - 90, 2012.

40. Duarte R. et al. Recomendações da Sociedade Portuguesa de Diabetologia para o Tratamento da Hiperglicemia e Factores de Risco na Diabetes Tipo 2. Revista Portuguesa de Diabetes, v. 2, n. 4, p. 5-18, 2007.

41. Geloneze Neto B. Perfil terapêutico dos incretinomiméticos. Revista Brasileira de Medicina, n. 3, p. 4-7, 2007. Suplemento Especial.

42. Mu J. et al, Chronic Inhibition of Dipeptidyl Peptidase-4 With a Sitagliptin Analog Preserves Pancreatic $\beta$-Cell Mass and Function in a Rodent Model of Type 2 Diabetes. Diabetes, v. 55, p. 1695-1704, jun. 2006.

43. Mancini MC, Halpern A. Aspectos fisiológicos do balanço energético. Arquivos Brasileiros de Endocrinologia \& Metabologia, São Paulo, v. 49, n. 3, jun. 2002.

44. Alfonso JEF, Ariza IDS. Nuevas terapias en diabetes: más allá de la insulina inyectable y de los antidiabéticos orales. Revista da Associação Médica Brasileira, v. 54, n. 5, p. 447-454, 2008.

45. Vieja ILB. Nuevas opciones terapeúticas para el tratamiento de la Diabetes Mellitus Tipo 2. Cuadernos del Hospital de Clinicas, v. 50, n. 2, p.74-83, 2005.

Nota: Artigo elaborado a partir da Monografia Final do Curso de Ciências Farmacêuticas, da Universidade Estadual de Feira de Santana (UEFS). 